\title{
ENABLING AIR-GROUND INTEGRATION: CONCEPT DEFINITION FOR TRAFFIC INFORMATION SERVICE IN CONTRACT MODE (TIS-C)
}

\author{
Rudi Ehrmanntraut, EUROCONTROL Experimental Centre (EEC), TALIS Project, \\ 91222 Brétigny sur Orge, France, rudiger.ehrmanntraut@eurocontrol.int
}

\begin{abstract}
The goal of this paper is to introduce the new TIS-C concept, and to give a high-level definition as well as to discuss its performance parameters. It was shown in the study "Alternative Enablers for Airborne Separation Assistance Systems (ASAS)" $[1]^{1}$ that Traffic Information Service in Contract mode (TIS-C) when compared with its broadcast counterpart TIS-B is a better candidate to support ASAS applications. That viewpoint was systematically developed, and a high number of arguments were developed to give evidence. Based on these findings it would be useful to give a better definition of the new TIS-C concept. .

The objective of TIS-C is to provide the flight deck with many kinds of traffic related information, using point-to-point telecommunications. The traffic information may be manifold and concern the ownship as well as its surroundings. The entire scope of the concept will be described by indicating the variety of possible information that can be presented in the flight deck, from the simple positions of other aircraft, to flight vectors, flight plans, trajectories, to airspace information and airport information.
\end{abstract}

Then some of the basic data used in TIS-C: position, flight plan, trajectory, and medium-term conflict will be described. The highest-level use cases of TIS-C will be defined. A first draft of the protocols that will be used between the TIS consumer and provider will be given: a Total Information Sharing Protocol. The architecture of TIS-C, will be described and its sub-enablers discussed.

Finally there is a conclusion with a number of steps to take if the TIS-C concept is to be brought towards implementation and an extrapolation of

\footnotetext{
${ }^{1}$ All references to conceptual documents from EURCONTROL, FAA, RTCA, NASA and EEC etc. are given in this study and are not repeated here.
}

future applications is given that could be provided with TIS-C.

\section{Introduction}

The integration of air and ground for Air Traffic Management is progressing, pushed by operational concepts of Co-Pperative Air Traffic Services and Collaborative Decision-Making. This trend raises a high number of new requirements concerning data availability in the flight deck. This paper gives a definition of the Traffic Information Services - Contract (TIS-C) concept that enables the flight deck to receive all manner of trafficrelated information to increase pilot situational awareness. Figure 1 shows the categorisation of TIS.

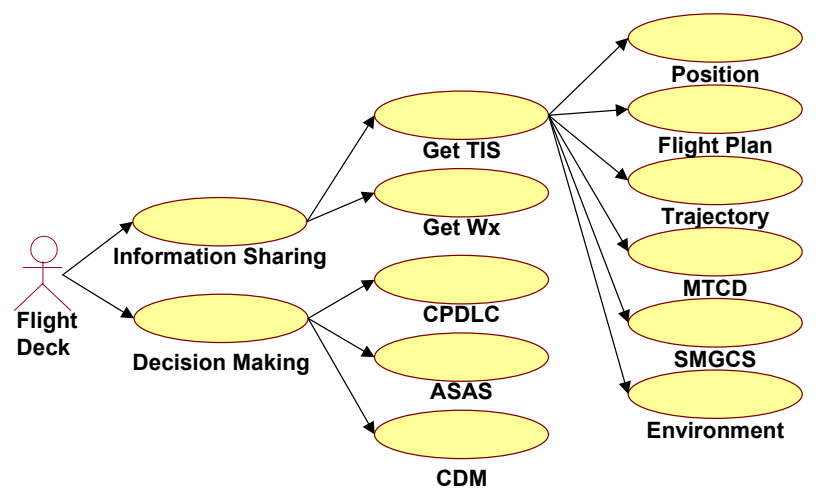

Figure 1. Categorisation of TIS

On the flight deck there will be two sorts of applications, information-sharing- and decisionmaking applications. TIS-C falls into the first category by providing the flight deck with information about its own and other aircraft: position, flight plan, trajectory, medium-term conflict, surface movement guidance and control, and environment data.

A potential application is shown in Figure 2, where the flight deck is alerted by a ground-based MTCD (Medium Term Conflict Detection) tool about a medium-term conflict, via TIS-C. TIS-C 
will make other traffic data available in an intelligent way, e.g. the trajectories or flight plans of aircraft that will be in the conflict area. The application could then predict the situation of the conflict at the time of conflict, as shown in a section below.

The following section defines the concept of TIS-C.

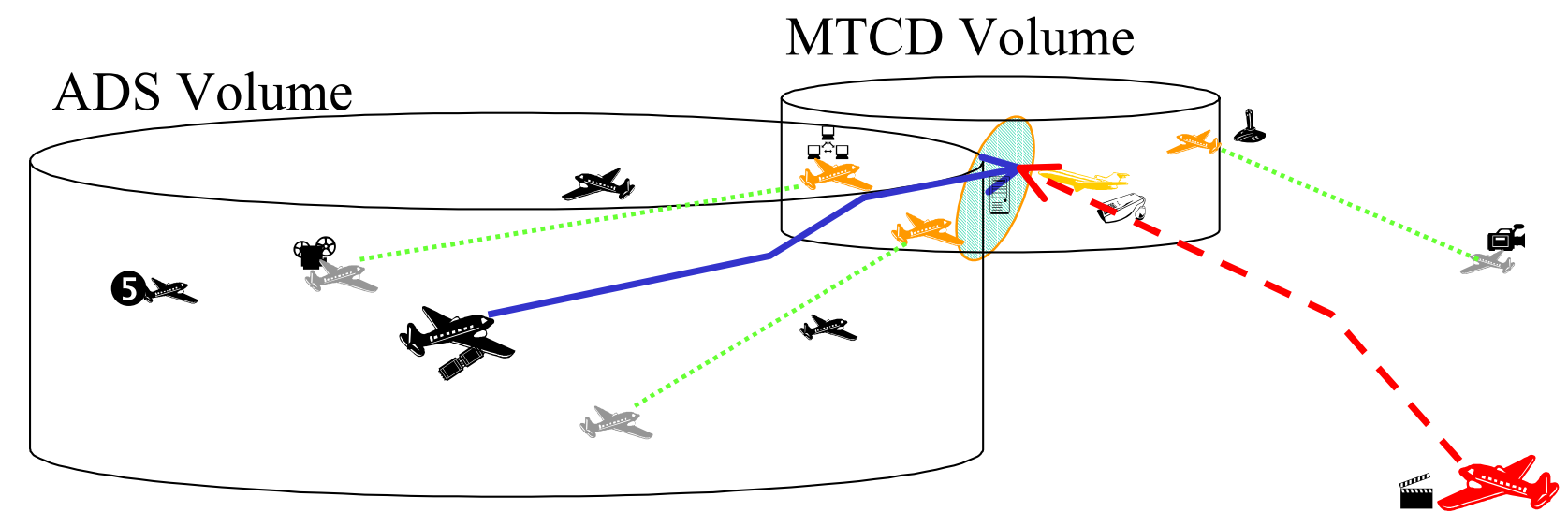

Figure 2. Illustrates An Example Of How TIS-C Can Provide Information To The Flight-Deck In Case of MTCD: The ownship gets position data in a volume of interest around the current position; if an alert occurs, then the position, flight plan or trajectory of the aircraft in conflict and also of other aircraft that will be in the conflict area are provided by TIS-C.

\section{Discussion}

\section{TIS-C Concept Definition}

Vision. Traffic Information Services are an enabler of CPDLC, ASAS and ADS-B operational applications and provide the pilot with information about other flights and their environment. The pilot uses this information for his/her situational awareness e.g. in a CPDLC environment where less information transits via radiotelephony. The pilot requires information when involved in airborne separation procedures, e.g. when tasks are delegated to the flight deck to separate it from leading or crossing traffic.

Context. TIS-C relates to CPDLC, ADS-B and TIS-B. In the study "About Alternative Enablers for ASAS" [1] it was shown that TIS-C has benefits in comparison to TIS-B, provided that there are mandated minimal solutions for ADS-B available, as is the case with Mode-S Extended Squitter. However, the "market" of TIS-C is bigger in comparison to TIS-B and ADS-B concepts because it can handle much more information, not being dependent on customised broadcast technologies.
Scope. TIS-C is targeted at airspace with CPDLC operations and ASAS operations. TIS-C covers gate-to-gate operations. TIS-C is targeted at commercial flight operations (in Europe) unless enabling technology becomes more affordable for other airspace users like General Aviation.

The time horizon for implementation of TIS-C is linked to the introduction of CPDLC and ASAS; probably on a voluntary basis for CPDLC operations and eventually a mandatory basis for some ASAS application categories. As development of TIS-C started only recently, it may not be available at the first introduction stages of CPDLC and ASAS, which are planned for about 2007-2010.

TIS-C gives information about traffic to the pilot including surrounding traffic positions, flight plans, trajectories, Medium Term Conflict Alert, Conflicting Aircraft Forecast, Surface Movement Guidance and Control (SMGCS) functions, environment information like airspace status etc. Weather and other applications like CDM (Collaborative Decision Making), CPDLC and ASAS clearances are not directly part of TIS-C. 
Goal. The goal of TIS-C is to increase pilot situational awareness concerning traffic-related information. Enhanced pilot situational awareness enables the flight deck to participate safely in CPDLC and ASAS operations.

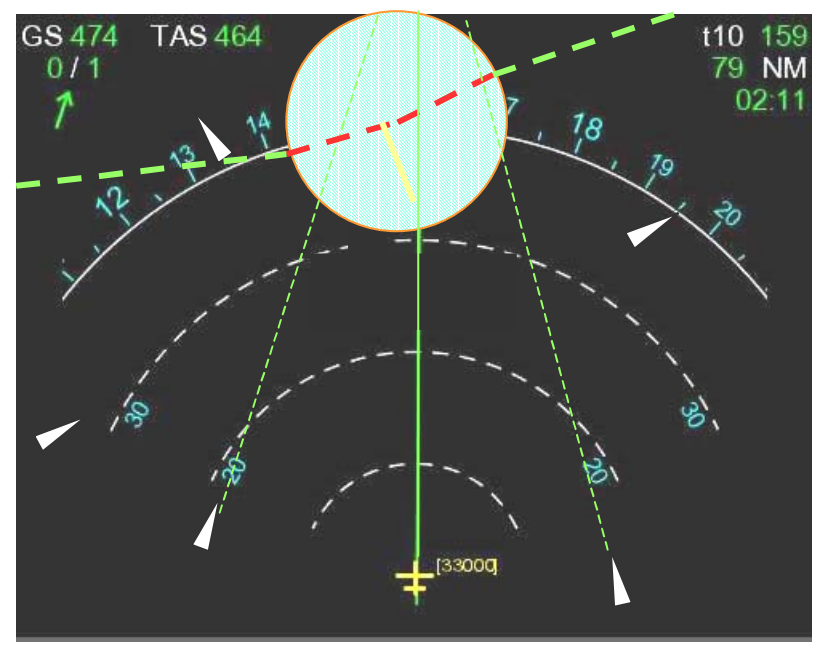

Figure 3. Shows A Medium-Term Conflict From The Perspective Of The Flight Deck. Even if the conflicting aircraft is not in the zoom area, i.e. the current volume of interest, its data is still available.

Outline of Concept. TIS-C is a concept where the flight deck is provided with many kinds of traffic related information by the ground using point-to-point data links.

TIS-C is a layered concept; i.e. it is composed of a technology-independent and a technologyrelated component. TIS ${ }^{2}$ (no B, no C) is technology independent and states the ATM requirements for traffic information for situational awareness. TIS-C is linked to a CNS (Communications, Navigation and Surveillance) technology and indicates that the traffic information is provided via a contract, and requires that this is enabled with a point-to-point telecommunications link. One layer affects the other in that the functions that can be provided on the operational layer are directly dependent on the underlying CNS capabilities, due to performance limitations of the air-ground data link.

TIS-C is a client-server application where the flight deck is the client that retrieves information from ground-based data servers. The client and the

${ }^{2}$ We do not mention the TIS from Mode-S, which is close to TIS-C with very limited functions. server must respect a common protocol. TIS-C establishes a contract between the client and the server, to which both must comply. The client may end that contract, but not the service provider. The contract also includes safe and seamless hand-over between service providers, e.g. in case the aircraft reaches the geographical limits of one service provider and is handed over to an adjacent one.

The information that is handled by TIS-C is related to traffic in the widest sense, and includes early definitions of aircraft position, flight plan, trajectory, medium-term conflict, environment data and airport surface movement and guidance data. The flight deck subscribes to receive traffic information in traffic volumes, which are like cylinders around the position of interest ,normally own ship.

Benefits. In this brief paper only a high-level statement can be given on the performance dimensions of TIS-C. TIS-C has safety benefits in that it provides situational awareness for traffic on the flight deck. The safety benefit is directly proportional to the fleet equipage rate and does not depend on population effects. TIS-C does not increase capacity by itself, but is an enabler for capacity increase with CPDLC and ASAS. TIS-C may increase security through shared situational awareness and vigilance, but may also do the contrary if it provides information to intruders of the system. TIS-C has no impact on environment, unless it is combined with e.g. the Weather ${ }^{3}$ service. Economically TIS-C will need a high investment, hopefully shared with other applications, and will possibly have a late return-on-investment.

Cost. TIS-C is an enabler concept that brings cost with it, which can be split into the investment cost and the operating cost. The components on which TIS-C is built need to be in place, i.e. datalink, CDTI in the flight deck, interconnected data servers and service providers on the ground. The operating cost is mainly the air-ground datalink cost, provision of data on the ground, and the maintenance of the entire equipment. The study [1] has argued that this cost is still less than TIS-B, because of possible cost share with other

\footnotetext{
${ }^{3}$ The second application that is developed in the TALIS 1 project.
} 
applications, its scalability and the use of the available frequency spectrum.

Sub-Enablers. TIS-C being an enabler for CPDLC and ASAS is itself dependent on three major sub-enablers or system components.

- Avionics: The flight deck needs to be equipped with graphical user interfaces like a CDTI and vertical display etc., and should link an input device e.g. the keyboard on the MCDU, to the screens. Higher integration with the FMS (Flight Management System) might be very useful to avoid wrong input by the pilots and enable faster response times..

- Data link: The aircraft must be equipped with at least VDL-2 (VHF Digital Link Mode 2), or in the future more powerful datalinks.

- Consistent flight data: The TIS-C service providers must provide consistent flight data. This gives strong requirements for system-wide information management.

- Transition. TIS-C has no specific transition problems. ANSPs (Air Navigation Service Providers) could provide the traffic services once aircraft are equipped. When Europe evolves towards higher integrated flight data management the service could be provided from a central entity.

- TIS-C Scenario

- Gate-to-Gate. The TIS-C concept can be illustrated with a scenario from gate to gate.

- Gate: The flight deck receives position information about neighbouring aircraft, possibly mixed with CDM and aircraft pushback orders.

- Surface: The flight deck receives position and vector information about surrounding aircraft and vehicles, possibly enriched with SMGCS guidance signals for taxi routing.

- Take-off and Climb: The flight deck receives position and velocity data for aircraft in a high cylinder within a small range, at a high update rate.

- En-route: The flight deck receives position information in a large and flat volume with low update rate.

- En-route CPDLC: Upon the event of a CPDLC clearance in proximity of the own ship, the flight deck receives traffic information of this aircraft, with the CPDLC clearance.

- En-route ASAS: The air traffic controller wishes to apply a co-operative procedure like station-keeping on another aircraft. The controller pushes traffic information with a contract for the lead-aircraft only, with its flight plan and trajectory, at a high update rate for position information.

- En-route Conflict: Upon a medium-term conflict, the flight deck receives, if possible trajectory data, otherwise flight plan data of all aircraft that will be in the predicted conflict area, with position data of other aircraft at time of closest point of approach. ${ }^{4}$ In addition it receives positions of other aircraft in a small volume around its position at a high update rate.

- En-route Weather Avoidance: If weather has to be avoided, then the same applies as for MTCD to make the flight deck aware of the other traffic that has to be re-routed as well.

- En-route: The flight deck receives environment information for sector information, Flexible Use of Airspace etc.

- Approach and Landing: The flight deck receives traffic information in a high cylinder with smaller range, possibly combined with airport CDM information and CPDLC services for runway use and visual range etc.

\section{TIS-C Architecture}

The architecture of TIS-C is based on a clientserver model with publish-subscribe event handling i.e. the client, who is the flight deck, subscribes to ground service providers.

\footnotetext{
${ }^{4}$ These are measures to optimise air-ground bandwidth, in an ideal environment all position, flight plans and trajectories will always be sent to the flight deck.
} 


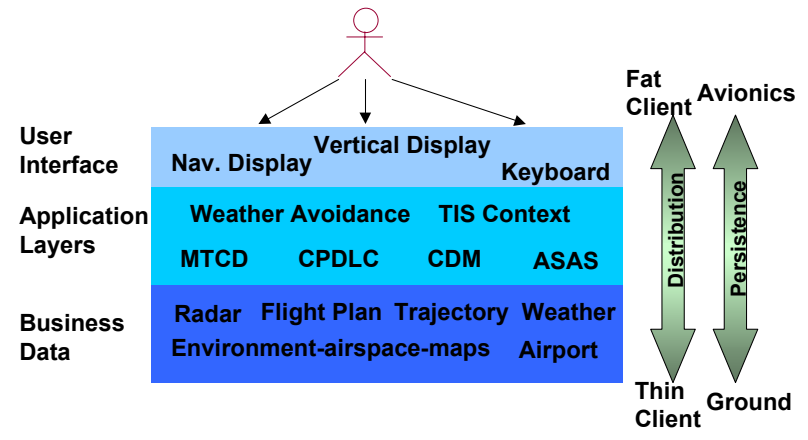

Figure 4. Illustrates the Layered Architecture Of TALIS Applications. It should be noted that TIS-C is mainly about data distribution and less an application in itself, yet it is put into the schema to show flight context dependencies.

TIS-C does not impose the architecture for client "intelligence" i.e. whether it serves as fat or thin client multi-tier architecture, nor for data persistency i.e. whether data is stored on the flight deck or not. Some functions are duplicated on the air and the ground e.g. it will make sense to include an ADS data tracker in the aircraft in addition to the one on the ground. The same applies for the MTCD algorithm that is allocated in the aircraft to work autonomously with ADS-B data, and on the ground.

Theoretically speaking, TIS-C is not a component in itself but falls into the category of software Connectors [2] that distribute data and events between components. This means that distribution is not an application in itself, it only enables distributed applications.

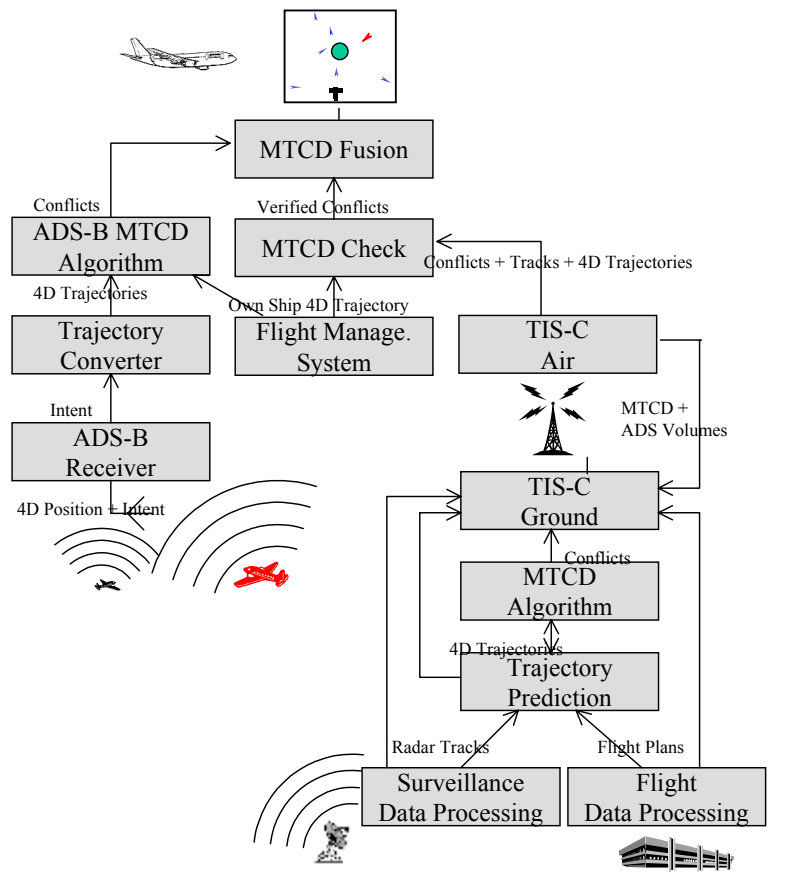

Figure 5. MTCD Functional Architecture Example

\section{TIS-C Use Cases}

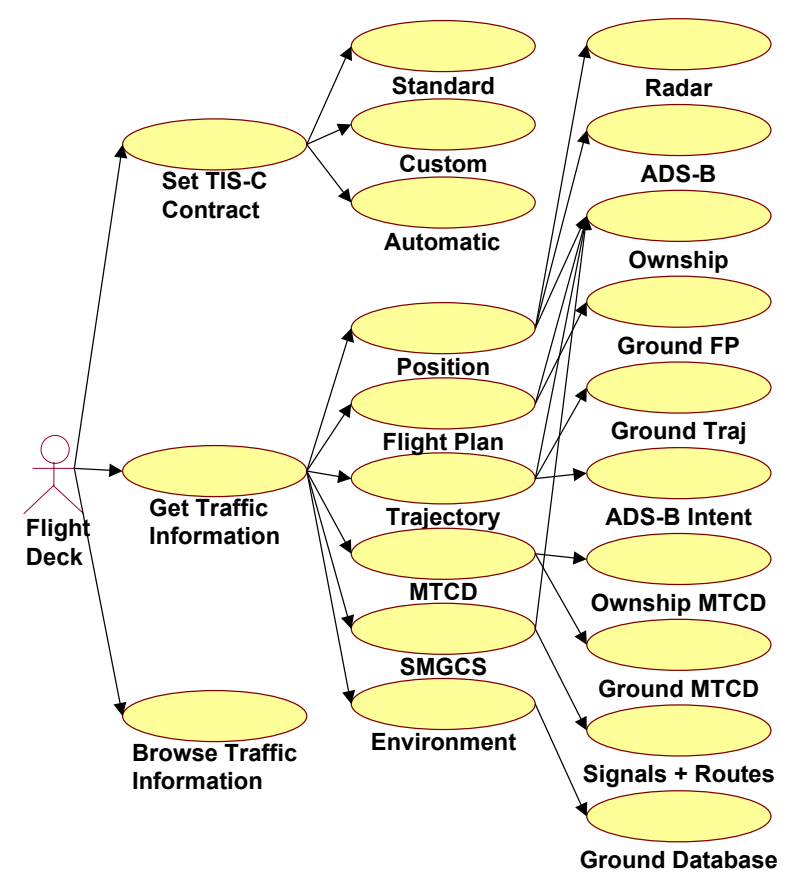

Figure 6. Shows the Three Highest-Level Use Cases With A Detailed Breakdown For "Get

Traffic Information". The right column indicates the data sources, where the MTCD of the ownship

is a client to TIS-C positions, flight plans and trajectories (which is not shown here). 
Use-Case Get Traffic Information is the central use case of TIS-C and gives traffic information to the flight deck and other applications on the flight deck, e.g. the avionics MTCD. For optimisation and accuracy it will always use the own ship data, ADS$\mathrm{B}$ data when available, and ground based data.

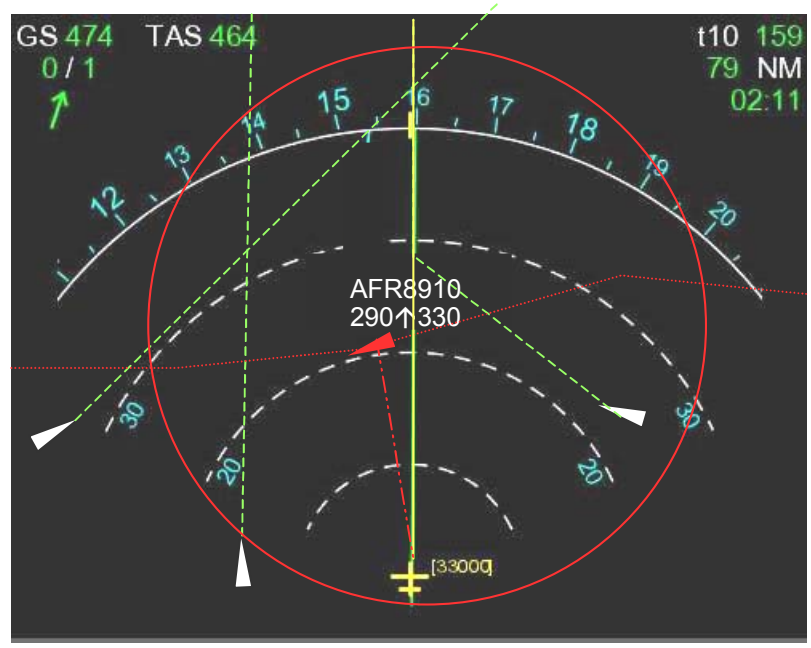

Figure 7. Shows that the Pilot Can "Browse" on the Conflict to Get the Predicted Situation of the Conflict Area. Information about other flights can also be shown with other user interfaces, like the vertical display and the MCDU screen.

Use-Case Browse Traffic Info allows the pilot to browse through the traffic information. The requirement is not to overload the pilot with information, but make it available either automatically for predefined situations, or to let the pilot browse for more information by selecting objects on the user interfaces and requesting more information about them. E.g. at any time the pilot may select a surrounding aircraft and request flight plan, trajectory, destination airport, runway etc.

Use-Case Set TIS-C Contract lets the pilots define the details of a TIS-C contract. As explained below there are several types of contracts predefined standard contracts and customised contracts. In addition the avionics might make automatic modifications of the contract, depending on its context, to optimise contracts for flight phases. This use case is for the settings of the standard and automatic contracts, as well as to input parameters for the customised contract.

\section{TIS-C Application Data Requirements}

When possible the data dictionary is derived from the AVENUE project [6] data definitions, which should be refined for the optimisation of the air-ground link.

\begin{tabular}{|l|l|l|}
\hline \multicolumn{3}{|l|}{ Air situation picture } \\
\hline 1 & Sequence of Track & Air situation picture \\
\hline
\end{tabular}

The position data is a subset of Asterix 62 [7] used for TIS-B. For further optimisation only trackelements 1,2,3,5 could be used.

\begin{tabular}{|l|l|l|}
\hline \multicolumn{2}{|l|}{ Track } & Flight ID \\
\hline 1 & FlightID & Track ID \\
\hline 2 & Integer & Position \\
\hline 3 & LatLong4DPosition & $\begin{array}{l}\text { - Heading } \\
\text { - Vertical Rate }\end{array}$ \\
\hline 5 & $\begin{array}{l}\text { - GroundSpeed } \\
\text { - TrackAngle }\end{array}$ & Ground Vector \\
\hline 6 & Integer & Data Source Identifier \\
\hline 7 & $\begin{array}{l}\text { Sequence of } \\
\text { LatLong4DPosition }\end{array}$ & Projected Profile \\
\hline
\end{tabular}

\begin{tabular}{|l|l|l|}
\hline \multicolumn{2}{|l|}{ Flight Plan } \\
\hline 1 & FlightID & Flight ID \\
\hline 2 & - NbOfAircraft & AircraftInfo \\
& - ICAOAircraftType & \\
& - AircraftTurbulence & \\
& - Model & \\
& - Company & \\
& - EngineModel & \\
& - FircraftType & \\
& - ICAOFhtType & \\
& - RawAcTypeZ & \\
\hline 3 & - AvionicData & \\
& - AirportName & DepartureInfo \\
& Block Day and & \\
& Time (EOBD, & \\
& - EOBT) & \\
& - ATD (estimated) & \\
\hline 4 & - AirportName & ArrivalInfo \\
& - ETA & \\
& - ATA & \\
\hline 5 & - AlternativeAirport & \\
\hline
\end{tabular}




\begin{tabular}{|l|l|l|}
\hline 6 & FlightLevel & RequestedFL \\
\hline 7 & Speed & CruiseSpeed \\
\hline 8 & $\begin{array}{l}\text { Sequence of } \\
\text { LatLong4DPosition }\end{array}$ & Route \\
\hline
\end{tabular}

\begin{tabular}{|l|l|l|}
\hline \multicolumn{3}{|l|}{ PredictedTrajectory } \\
\hline 1 & FlightID & Flight ID \\
\hline 2 & $\begin{array}{l}\text { Sequence of } \\
\text { LatLong4DPosition }\end{array}$ & PredictedTrajectory \\
\hline
\end{tabular}

\begin{tabular}{|l|l|l|}
\hline \multicolumn{3}{|l|}{ Medium-Term Conflict List } \\
\hline 1 & $\begin{array}{l}\text { Sequence of } \\
\text { MediumTermConflict }\end{array}$ & MTCD List \\
\hline
\end{tabular}

\begin{tabular}{|l|l|l|}
\hline \multicolumn{3}{|l|}{ MediumTermConflict } \\
\hline 1 & CoflictID & MTCD ID \\
\hline 2 & $\begin{array}{l}\text { CPATime } \\
\text { (mandatory), } \\
\text { StartTime/EndTime } \\
\text { (optional) }\end{array}$ & ConflictTime \\
\hline 3 & $\begin{array}{l}\text { - WakeTurbulenceCa } \\
\text { tegory } \\
\text { - CoflictGeometry }\end{array}$ & OtherFlight \\
\hline 4 & CoflictGeometry & OwnShip \\
\hline 5 & $\begin{array}{l}\text { Sequence of } \\
\text { - PositionCPATime } \\
\text { - PredictedTrajectory }\end{array}$ & $\begin{array}{l}\text { AircraftInConflictAre } \\
\text { a }\end{array}$ \\
\hline
\end{tabular}

\begin{tabular}{|l|l|l|}
\hline \multicolumn{3}{|l|}{ CoflictGeometry } \\
\hline 1 & FlightID & Flight ID \\
\hline 2 & LatLong3Dposition & StartOfConflict \\
\hline 4 & LatLong3Dposition & $\begin{array}{l}\text { Closest Point Of } \\
\text { Approach (CPA) }\end{array}$ \\
\hline 4 & LatLong3Dposition & EndOfConflict \\
\hline
\end{tabular}

ATM Environment. Not yet defined. Airport. Not yet defined.

Ad. MTCD. In case of a MTCD it was decided to uplink the own ship conflict-geometry as well, even though the flight deck could compute it from the geometry data of the conflicting aircraft. This is to enable an additional check for the validity of the conflict. The own ship should confirm the conflict with its own trajectory information and compare the result with the given information. If there are 'significant' differences, then the aircraft should update the ground server with its own computed trajectory, and the conflict should disappear.
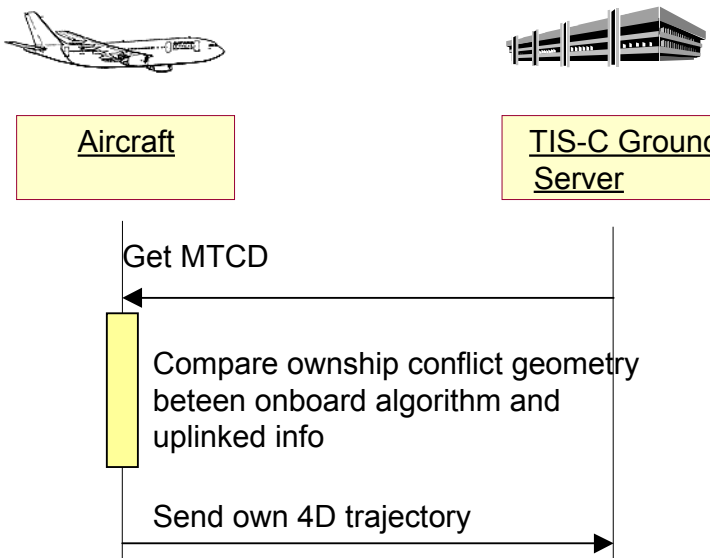

Figure 8. Is An Illustration That The Aircraft Uses the uplinked geometric conflict information concerning itself to verify the conflict, and in case of discrepancy updates the ground server with its own predicted trajectory computation.

\section{TIS-C Contracts}

The TALIS system is conceived to operate in an environment of service providers and service users, competition between service providers, and free choice of services for the service users. Therefore special attention has been put on the negotiation of contracts between service providers and service users. In addition the notion of third parties has been introduced so that contracts can be negotiated on behalf of a party, e.g. an airline negotiating a company contract for all of its aircraft, or ATC (Air Traffic Control) imposing standard contracts for all aircraft and service providers.

TIS-C contracts are defined between the flight deck and ground service providers for the delivery of traffic services. One aircraft can make one or more contracts with one or more service providers. Several contracts with the same service provider are useful to have a concatenation of data that leads to superposition for the flight deck. E.g. the flight deck makes one contract for position data in a large volume with a low update rate, another contract for position data in a small volume at a high update rate, and yet another contract specifying MTCD parameters. The aircraft can also make contracts with several service providers, e.g. to get en-route traffic information from a national traffic service provider, environmental data from a central 
European one, and airport information from a local airport service provider.

The contract defines all terms of service provision. It contains a number of paragraphs that are agreed upon. The paragraphs are specific to the service and shall be standardised for all mandatory paragraphs (parameters) or conform to a convention. The paragraphs of a contract are defined as follows:

- $\quad \S$ TIS Parties defines the roles and the power of the parties involved in TIS contract negotiation, and may be an entitled service user like the aircraft, AOC, ATC and airport; an entitled service provider; and an entitled third party like ATC and AOC.

- $\quad \S$ TISP Policy defines the policy that is applied for the TIS protocol as defined in a section below. It specifies which party is entitled to initiate a negotiation; which party is entitled to end a contract; which party is entitled to update a contract; which party is entitled to initiate the hand-over of the service; whether a standard or custom contract is allowed; and how many contracts may be concatenated. We suggest for TIS that all parties may initiate the negotiation; that all service users and their third parties may end a contract; that all parties may update and initiate a hand-over; that standard contracts are used; and that not more than 4 contracts can be concatenated.

- $\quad \S$ TIS Requested Information defines the type of information i.e. aircraft identification, 3D position, time, figure of merit, ground vector, air vector, projected profile, short term intent, intermediate intent and extended projected profile, flight plan whole or leg, or 4D trajectory whole or leg, and MTCD conflict data. In addition it defines data filters like the dimensions of the volume of interest with a parameter for range, relative flight level distance above and beneath; data filter to send only aircraft position for non-ADS-B aircraft; data filter to send only position for one aircraft; and data filter for aircraft in medium-term conflict.

- $\quad \S$ TIS Delivery Strategy defines when traffic information is provided, i.e. whether periodically, on demand, on event or on emergency. For TIS we consider the following parameter settings: Periodicity of $3,5,10,15,30$ seconds, above interval of 15 seconds typically 1, 5, 10, 15 minutes; On demand upon a user interaction; On event is more complex because four types of events are identified, where each event type can have parameters. The four event types are Severity, Information, Flow Management and ATC event.

- $\quad \S$ TIS Quality of Service defines the cost of service, the cost-sharing model for the telecommunications cost, and the granted figure of merit.

\section{TIS Standard and Company Contract. Traffic} Information in the flight deck enables ATC application for co-operative air traffic services. The main users are therefore ATC and the pilot. ATC being a safety critical application, the TIS contracts must comply with minimum performance requirements. Therefore it should be envisaged that TIS contracts are predefined by ATC and all involved parties must comply with the terms. Such a predefined contract is called a standard contract.

The same may apply to AOCs and service providers when they negotiate a contract with specific conditions, which are fixed in the company contract. When a user is searching for a service, it should use the service providers with which standard or company contracts exist. TIS for ATC should be based on standard contracts.

This summarises the current definition of the contract. The following section defines the protocol that is used between user and service provider.

\section{TIS-C Protocol Requirements}

The Total Information Sharing Protocol (TISP) is explained in the paper [3], and here is given just a brief summary of its features. TISP is a generic protocol for service discovery, service negotiation, service subscription, service delivery, and seamless service hand-over. TISP can be used by all information sharing applications that need to retrieve data from service providers, and that need to get contractual service delivery; therefore it is not limited to TIS-C. 


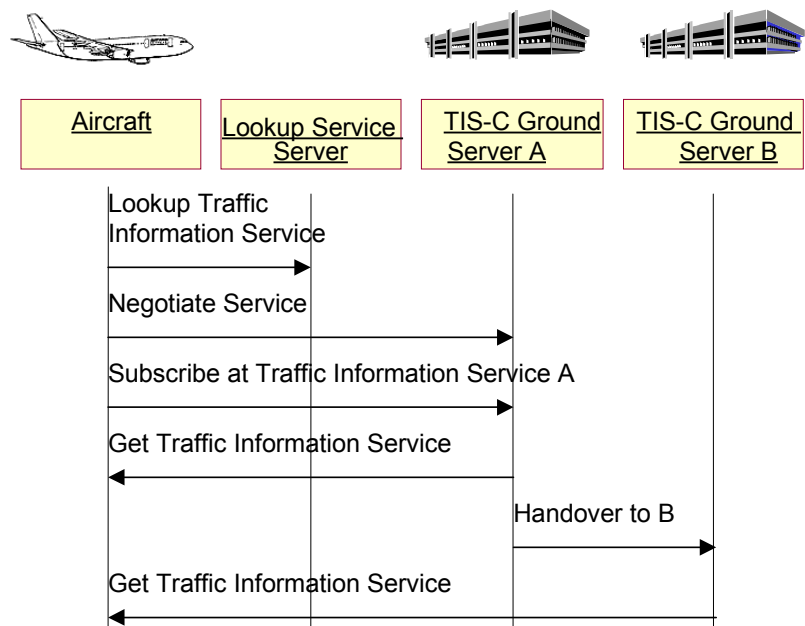

Figure 9. Shows The Simplified Total-InformationSharing-Protocol (TISP), a generic protocol for information sharing of mobile users, with service discovery, service negotiation, service subscription, service delivery, and seamless service hand-over.

TISP is discussed with respect to its atomic patterns.

Service Provider Discovery. Users find services through dynamic service discovery ${ }^{5}$.

Contract Negotiation. A contract is negotiated between the client and the service provider. A contract can also be negotiated between a third party on behalf of a client and a service provider, e.g. in the case that ATC wishes a specific aircraft to receive traffic information. A special case of contract negotiation is when the client or third party sends an existing contract identification, which refers to a previous or standard contract.

Service Subscription. The service user is subscribed to the service upon successful negotiation of a contract.

Service Delivery. Services are delivered to the users upon successful subscription to the service.

Service Update. The user or the service provider can make an update to the contract, to modify the current service delivery without going through an entire contract negotiation.

\footnotetext{
${ }^{5}$ In opposition to the ATN where users get service provider addresses through a distributed forward naming service (that we don't like) called Context Management.
}

Service Hand-Over. The hand-over to another service provider gives the possibility of seamless services to the user. The conditions of the handover are defined in the contract. The service provider must comply with these conditions. The service provider forwards the contract with one aircraft to another, probably an adjacent service provider. The next service provider must confirm the hand-over. The service hand-over must be synchronised so that no hole in service provision occurs.

Service End. Both sides can initiate the end of service. The conditions for the end of service are stated in the contract i.e. only the client or client party may end the contract for the TIS application, other applications may not have this contractual restriction. The reason for the end of service should be given when it is not at a hand-over, e.g., service not needed anymore, service boundary reached etc.

\section{Other Attractive Features}

There are some other attractive use-cases of TIS-C that wil be highlighted here.

Third Party. The negotiation and the subscription to a contract by a third party have been defined above. This very useful feature allows ATC or AOCs to push services for aircraft, without the pilot being the initiator. This is especially useful and economical for the probably cumbersome contract negotiation. This could, for example, result in the negotiation of a standard contract with a service provider for an entire flight region or parts of Europe. Very useful is the subscription to services on behalf of the user, e.g. when ATC wishes information to be pushed into the flight deck, or the ATCO wishes to apply station-keeping on one aircraft and pushes traffic information of the leading aircraft to the trailing aircraft.
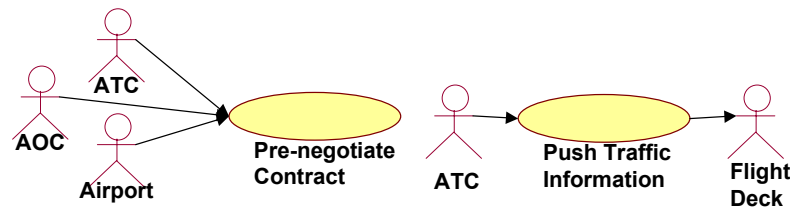

Figure 10. Use Cases For A Pre-Negotiation Of Contracts By Third Parties, and pushing data to the user by subscription of a third party to the service. 
Automatic Contract Modifications. The human users or machines may modify contracts. The modification of the contract by a machine may be very useful to optimise traffic information for the current context of the aircraft. This may vary from very basic modification like the adaptation of the volumes of interest for different flight phases and to adapt to own speed etc. up to more complex situational contexts like modifications of contracts when under specific procedures. For example the behaviour for MTCD as described above is a situation where flight information of other aircraft that will be in the conflict is also sent to the flight deck. Other scenarios can be conceived for the combination of specific event types with requests for specific information types. This subject is under investigation and the study [4] gives some first findings.

Reacting on Event Types. Four event types have been introduced: Severity-type, ATC-type, Flow-Management-type and Information-type events. Severity type examples are: MTCA, military special-use airspace (SUA) occupied, or significant weather change. An ATC type is when surrounding aircraft receive CPDLC clearances, or surrounding aircraft are transferred to another $\mathrm{R} / \mathrm{T}$ frequency etc. Examples of flow management types are; changes to airport departure and arrival lists, surrounding aircraft change from or to ASAS-mode, or weather changes that impact flow management, or availability of flexible airspace. Examples of information types are the updated trajectory from other aircraft, updated flight plans from other aircraft, updated civilian special-use airspace, or less significant weather changes. It is important to give some guidelines for use because the combination of types can become quite complex and lead to disorganised behaviour.

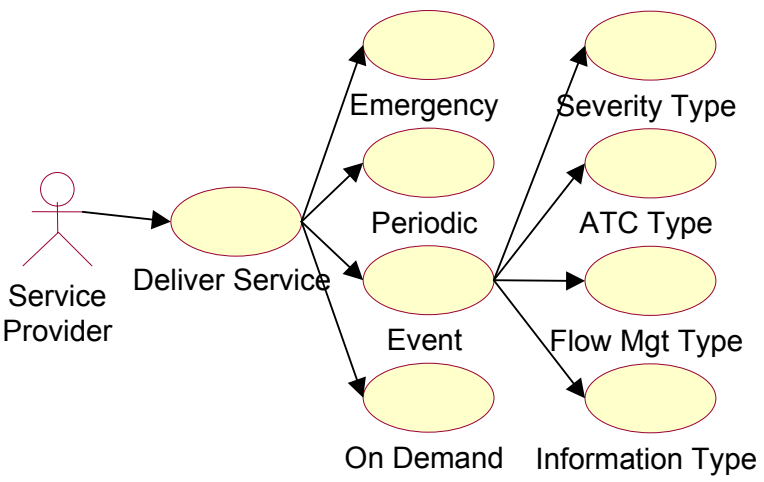

Figure 11. Shows The Use-Case For The Delivery Strategy Of The Service Provider, with the refined event types.

All aircraft should subscribe to severity-type events, at all times. Severity events should lead to a maximum of related information, e.g. SUA airspace events should lead to getting environment data plus trajectories of surrounding aircraft; severe weather changes should lead to weather updates and trajectories of involved aircraft as already explained for the MTCD event. All aircraft should subscribe to ATC-type events, but only in a limited volume. Aircraft may subscribe to flow-type events when under procedures for enhanced tactical flow like sequencing for airports or en-route station keeping. Aircraft should subscribe to traffic information in a large volume at low update rates to enhance situational awareness.

\section{TIS-C Sub-Enablers}

Avionics are needed for the treatment of different information by the flight deck, and where appropriate show it to the pilots. Therefore the cockpit should be fitted with a Navigation Display like a CDTI, and enhancements should be done for this. A vertical display is very useful for the visualisation of data of vertical interest as during climb and descent flight phases. The displays must be integrated with input devices like a keyboard, usually on the MCDU today, or other pointing input devices. Last but not least a high integration with CPDLC and FMS will help to update the ownship information.

Air-Ground Mobile Networks. The TIS-C concept is based on the availability and sufficient performance of mobile air-ground networks. This can already be achieved by the use of VDL 2 ; however, it can be foreseen that the performance of 
VDL 2 will not be sufficient if many aircraft use TIS-C. Research is necessary to develop networks with higher performances. Another mitigation strategy to work around the low performance of today's air-ground networks is to evaluate the risk of using commercial telecommunications as proposed for the cabin. That may work for some categories of non-critical data.

Consistent and intelligent data management is a cornerstone of this concept. There is a major safety risk if the data that is distributed is not accurate, consistent, available, interoperable, integrated, secure and safe. This needs some improvements in the European ATM system, which requires integration of Central Flow Management Unit, Air Traffic Service Providers, Airports, military control, airlines with their aircraft, or other independent aircraft. System-wide Information Management is required.

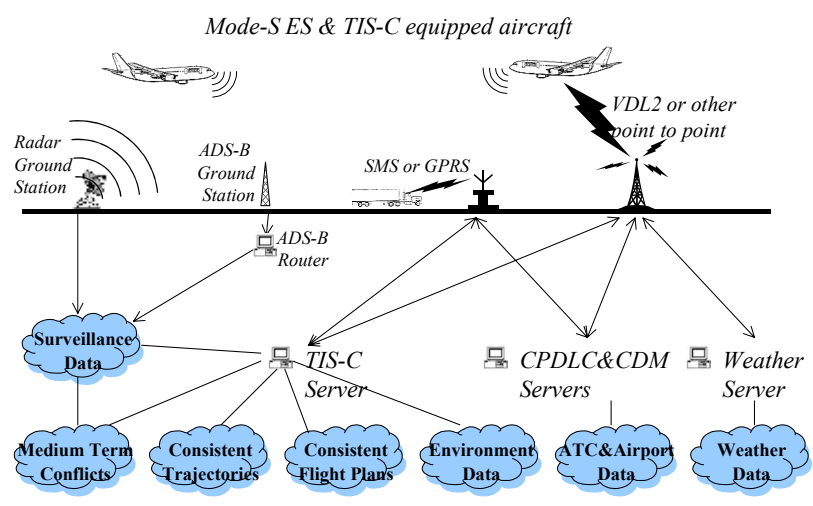

Figure 12. Illustrates That the InformationSharing Network Is Based on Several Data

Sources. These must be accurate, consistent, available, interoperable, integrated, secure and safe.

\section{TIS-C Future Evolution}

Towards Implementation. There will be a number of decision points before TIS-C can be implemented. Being an enabler for CPDLC and ASAS it also depends on the implementation of these. The ASAS community, which is closely linked with ADS-B and TIS-B, must be convinced that TIS-C has advantages in comparison with TIS$\mathrm{B}$ and the defenders of UAT and VDL 4.

Technically the TIS-C concept has to define its telecommunications performance, prove that it is feasible over VDL 2, and show the performance limits. Technically, TIS-C has to be validated, with a special focus on safety. Institutionally, TIS-C has to be standardised. Economically, TIS-C has clear benefits in comparison to TIS-B, yet both TIS -B or $-\mathrm{C}$ must show adequate returns on investment.

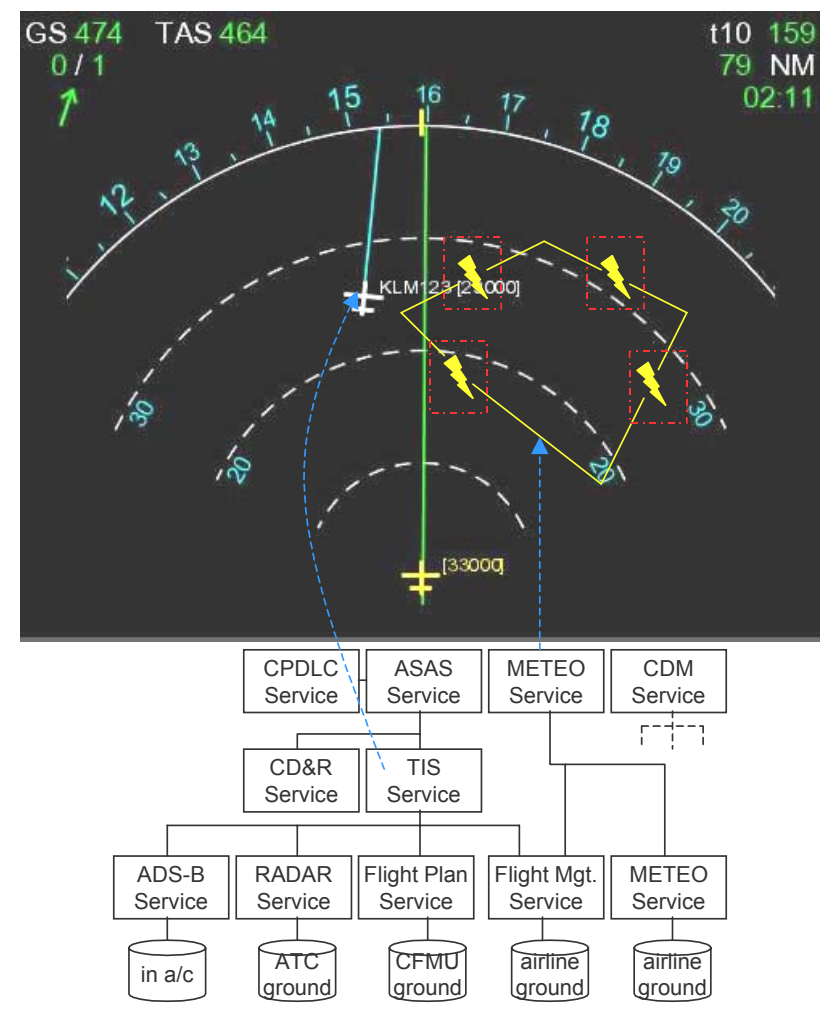

Figure 13. Illustrates The Prototype Cockpit From THALES Avionics As Used in the TALIS Project. In the future one could combine weatherand traffic information services with tactical-flowmanagement- and separation services.

New Applications. One of the features of TIS$\mathrm{C}$ in comparison to its competitors is that it is scalable and allows for new applications with minimum effort using the same initial infrastructure. If the trend towards co-operation in ATS continues, then it can be imagined that a high number of new and additional applications will develop next to and in TIS, because all information that is provided to the controller today will be needed on the flight deck tomorrow. Also other information like gate-management, passenger management etc. will be given to the flight deck in the right context. The TALIS project foresees a higher integration between the information-sharing part that is provided by TIS-C and the decision- 
making applications like CPDLC; ASAS and CDM. The arrival of more flow-related applications is foreseen, e.g. the severe-weather-avoidance-routing application shown in Fig. 12 that combines TIS, Weather, and tactical flow management, eventually enabled by station-keeping.

\section{The TALIS Project}

The TALIS (Total Information Sharing for Pilot Situational Awareness Enhanced by Intelligent Systems, http://talis.eurocontrol.fr) project develops specifications and prototypes for a distributed information-sharing system providing TIS-C and Weather services. The approach of the project is to focus on the overall integration of existing system components into a system of systems with the help of a Federation Architecture. This Federation Architecture will handle collaborative, coordinated, distributed, and consistent informationsharing and decision-making.

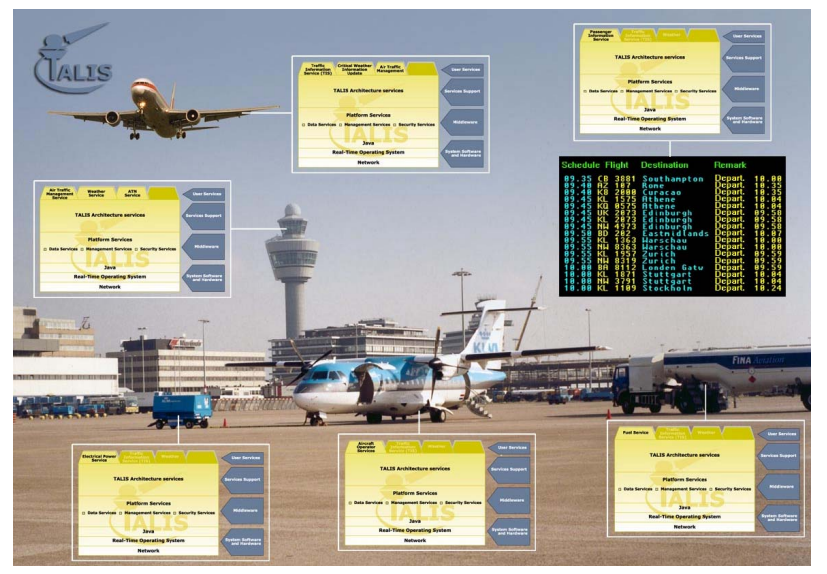

Figure 14. Illustrates the Vision of the TALIS

Project: A collaborative, distributed, interoperable, consistent, available and integrated information sharing system [5].

Requirement, specification and architecture documents for TIS-C will be available on the WWW soon. A demonstration is planned for early 2004.

\section{Conclusions}

This paper defined the concept of TIS-C, with a discussion of vision, context, scope, goal, outline of concept, cost, benefits, sub-enablers and transition. Further paragraphs have covered detail for most of these, with a special focus on the outline of the concept itself.

The solution that is presented is a contractbased protocol between the flight deck as a primary client and ground-based service providers. The paper arguments that it is important to base the relationship of service consumer and user on a contractual basis, primarily for safety reasons in a commercial environment. Furthermore the information that is distributed by TIS-C is defined: position, flight plan, trajectory, MTCD, but leave SMGCS and environmental data undefined at the moment. TIS-C needs to be based on a globally acknowledged protocol that will enable operations and interoperations world-wide.

The TALIS consortium is convinced that TIS$\mathrm{C}$ is the way forward for the support of CPDLC, ASAS and CDM, and any future concept like tactical flow management and integration with ground automation. The combination of these concepts in some powerful future applications for the flight deck has been demonstrated.

The R\&D part of the TIS-C concept is well advanced. Ongoing studies confirm its feasibility over VDL2 ${ }^{6}$. Decisions in favour of the concept are now needed for further elaboration and validation. TIS-C has cost-benefit advantages in comparison to TIS-B. The authors believe that nothing should really stop TIS-C.

\section{Acknowledgements}

This paper has been created for the TALIS project funded by the European Commission, DG IST, and the project partners LIDO, NLR, Skysoft, THALES Avionics and EEC.

We thank the European Commission officers, as well as stakeholders in EUROCONTROL for their support.

\footnotetext{
${ }^{6}$ Soon to come: Assessment of TIS-C over VDL2
} 


\section{References}

[1] Rudi Ehrmanntraut, Dec. 2002, Alternative

Enablers for ASAS, EUROCONTROL

Experimental Centre

[2] Rudi Ehrmanntraut, Air-Ground Connector

[3] Rudi Ehrmanntraut et al. , 2003, Towards a

Concept Definition of the Total Information

Sharing Protocol, EEC.

[4] Joseph Bauer, 2003, Identification and Modeling of Contexts For Different Information

Scenarios in Air Traffic, TU Berlin and EEC, Diplomarbeit.

[5] Ernst Kesseler et al., 2002, Integrating Navigation and Communication Systems for Innovative Services, NLR and EEC, in Proceedings of 9th St Petersburg Conference for Integrated Systems.

[6] AVENUE data dictionary, http://www.eurocontrol.fr/projects/avenue/

[7] EUROCONTROL, 2000, Standard Document for Surveillance Data Exchange Part 9, Transmission of System Track Data, SUR.ET1.ST05.2000-STD-09-01

\section{Acronyms}

ADS-B

Automatic Dependent Surveillance Broadcast

AOC Airline Operations and Control

ASAS Airborne Separation Assurance System

ATC Air Traffic Services

ATM Air Traffic Management

ATN Aeronautical Telecommunications Network

ATS Air Traffic Services

ATSU Air Traffic Service Unit, sometimes used as synonym for Air Navigation Service Provider (ANSP)

CDM Collaborative Decision Making

CDTI Cockpit Display for Traffic

Information
CNS

COOPATS

CPDLC

DG IST

EEC

GPRS

GSM

MCDU

MODE-S

Mode-S ES

MTCD

$\mathrm{R} / \mathrm{T}$

RWTH

SMGCS

SUA

TALIS

TIS

TIS-B

TIS-C

TISP

UAT

VDL

$\mathrm{Wx}$
Communications, Navigation and Surveillance

Co-operative ATS

Controller-Pilot Data Link Communications

Direction Generale - Information Society and Technology

EUROCONTROL Experimental Centre

General Packet Radio Service

Global System for Mobile Communication

Multi Cockpit Display Unit

Secondary Surveillance Radar MODE-S

Secondary Surveillance Radar Mode-S Extended Squitter

Medium Term Conflict Detection

Radiotelephony

Rheinisch-Westfälisch Technische Hochschule Aachen

Surface Movement Guidance \& Control

Special Use Airspace

Total Information Sharing for Pilot Situational Awareness Enhanced with Intelligent Systems

Traffic Information Service

TIS Broadcast

TIS Contract

Total Information Sharing Protocol

Universal Access Transceiver

Very High Frequency Digital Link

Weather 